\title{
Télévision et socialisation : continuité ou perturbation des mécanismes classiques de transmission des acquis culturels aux jeunes à Baco Djicoroni, Bamako
}

\section{Television and socialization: continuity or disruption of classic transmission mechanisms of cultural achievements for young people in Baco Djicoroni, Bamako}

Sangho $F^{1^{*}}$, Sangho $A^{2}$, Sangho $F^{3,4,5}$, Sangho $\mathrm{O}^{3,5,6}$, Touré $F^{7}$, Diallo $B^{8}$, Coulibaly $C A^{5}$, Telly $N^{5}$, Kéïta $S^{5}$, Togo $Y^{5}$, Oumar $Y^{6}$, Sissoko $\mathrm{N}^{6}$, Seydou $\mathrm{F}^{9}$, Sangho I ${ }^{10}$, Togola OB ${ }^{11}$, Dansoko DD ${ }^{5}$, Camara B ${ }^{6}$, Somboro AH ${ }^{6}$, Coulibaly M ${ }^{6}$, Diakon B ${ }^{12}$

\section{DOI : 10.53318/msp.v11i1.1891}

1. Cellule de Planification et de Statistique, Secteurs Santé, Développement Social et Promotion de la Famille (CPS/SSDS-PF), Bamako, Mali

2. Département d'Enseignement et de Recherche (DER) des Sciences Pharmaceutiques, Faculté de Pharmacie (FAPH), Université des Sciences, des Techniques et des Technologies de Bamako (USTTB), Mali

3. DER des Sciences Biologiques et Médicales, FAPH / USTTB Mali

4. Direction de la Pharmacie et du Médicament (DPM), Bamako, Mali

5. DER en Santé Publique et Spécialités (DERSP), Faculté de Médecine et d'Odontostomatologie (FMOS), USTTB, Mali

6. Agence Nationale de Télésanté et d'Informatique Médicale (ANTIM), Bamako, Mali

7. Programme National de Lutte contre le Paludisme (PNLP), Mali

8. Centre Hospitalier Universitaire Odonto-Stomatologie (CHUCNOS), Bamako, Mali

9. Population Council Services (PSI), Bamako, Mali

10. Observatoire pour les Elections et la Bonne Gouvernance, Bamako, Mali

11. Sous-direction Prévention et lutte contre la maladie de la Direction Générale de la Santé et de l'Hygiène Publique, Bamako, Mali

12. DER de Sociologie Anthropologie, Faculté des Sciences Humaines et des Sciences de l'Éducation, Université des Lettres et des Sciences Humaines de Bamako

*Auteure Correspondante : Fadimata SANGHO, Fonctionnaire d'Etat à la Cellule de Planification et de Statistique secteurs Santé, Développement social et Promotion de la Famille (CPS/SS-DS-PF), Tél : +223 763762 33, Email : fadimasangho@gmail.com.

\section{Résumé :}

Introduction : L'objectif principal était de comprendre l'influence de la télévision sur les mécanismes classiques de transmissions des acquis culturels aux jeunes. Méthodes : Nous avons procédé à l'interview de 8 parents, 13 adolescents (dont 9 filles) et 4 spécialistes de l'éducation à Baco-Djicoroni. Nous avons aussi mené des entretiens avec des responsables de programmes et d'émissions de l'ORTM. Une analyse de contenu a été réalisée sur les données qualitatives collectées. Microsoft Excel a été utilisé pour l'analyse quantitative. Résultats : Les personnes interrogées avaient un âge compris entre 30 à 50 ans (parents) et 12 à 15 ans (adolescents) pour la majorité. Tous les parents et adolescents ont affirmé avoir la télévision en famille à l'exception d'un parent qui avait plus de 50 ans. Elle était regardée en famille par la plupart des enquêtés. Les parents pensent que la télévision pérennise les valeurs culturelles tandis que les spécialistes de l'éducation $(n=4)$ la conçoivent comme un outil perturbateur de la transmission des acquis culturels.
Quant aux responsables des programmes et émissions de l'ORTM, ils affirment que la chaîne nationale propose des émissions participant à la transmission des valeurs culturelles aux jeunes. Aussi, la moitié d'entre eux pensent que ce médium assure la continuité des mécanismes classiques de transmission des acquis culturels. Conclusion : Les programmes de télévision doivent être revus pour influencer positivement la socialisation de la jeune génération et assurer la transmission des acquis culturels.

Mots clés : Télévision, socialisation, culturel, jeunes, Baco-djicoroni.

\begin{abstract}
:
Introduction: The main objective was to understand the influence of television on conventional transmission mechanisms cultural achievements for young people. Methods: We interviewed 8 parents (including 2 women), 13 teenagers (including 9 girls) and 4 education specialists in Baco -Djicoroni. We also conducted interviews with ORTM program and program managers. These interviews were supplemented by the literature review. A content analysis was carried out on the qualitative data collected. Microsoft Excel was used for quantitative analysis. Results: Most respondents were between 30 and 50 years of age (parents) and 12 to 15 years of age (adolescents). All parents and teens reported having TV in their families except for one parent who was over 50 years of age. It was watched as a family by most of the interviewees. Parents believe that television perpetuates cultural values while educators $(n=4)$ view it as a disruptive tool for the transmission of cultural achievements. ORTM's program and program managers stated that the national channel offers programs that help to transmit cultural values. Thus, half of them believe that this medium ensures the continuity of the traditional mechanisms of transmission of cultural achievements. Conclusion: Television programs should be reviewed to positively influence the socialization of the younger generation and to ensure the transmission of cultural achievements to young people.
\end{abstract}

Keywords: Television, socialization, cultural, youth, Baco -Djicoroni.

\section{Introduction}

La socialisation désigne les mécanismes de transmission de la culture ainsi que la manière dont les individus reçoivent cette transmission et intériorisent les valeurs, les normes et les rôles qui régissent le fonctionnement de 
la vie sociale (1). La socialisation doit être considérée comme un processus continu qui concerne les individus tout au long de leur vie (2). On distingue classiquement une socialisation primaire et une socialisation secondaire (2). La socialisation primaire correspond à la période de l'enfance (3). Ce processus s'effectue d'abord dans la famille qui en constitue l'instance principale ; son action est essentielle pour la structuration de l'identité sociale (3). L'école représente une autre instance majeure de la socialisation primaire : pour Émile Durkheim, cette socialisation méthodique de la jeune génération par la génération adulte permet d'inculquer les normes et les valeurs qui constituent le fond commun de la société $(3,4)$. Le moteur de toute société réside dans la bonne éducation de sa jeunesse de laquelle dépend l'avenir. Plusieurs analyses du mode actuel sur le plan culturel font état de l'échec de la transmission des valeurs culturelles d'une génération à l'autre. La thèse qui soutient cette peur bien connue des sociologues et historiens de la culture s'oppose à celle qui considère les pratiques et comportements des jeunes d'aujourd'hui comme les manifestations d'une appropriation des avancées technologiques du monde (5). McCoy trouve des facteurs influençant la socialisation des enfants tels que la télévision et le rôle de la famille (6). Atkin et Yoo identifient d'autres facteurs comme l'ethnie et la race (7). Des facteurs socio-culturels ont été aussi cités (8).

La télévision peut être définie comme l'ensemble des activités et des services assurant l'élaboration et la diffusion d'informations et de spectacles, des programmes, etc. (Émissions, programmes, studios, régie, chaînes de télévision) (9). Les développements des grands moyens de diffusions favorisent l'émergence de la communication de masse, et influencent de ce fait tous les domaines de la vie humaine et sociale. Ces moyens de communications sont de sérieux concurrents d'institutions comme la famille, l'école et la religion sur le plan de l'éducation. L'influence des médias modernes tels que la télévision, les réseaux sociaux qui mettent en mal les mécanismes de socialisation des jeunes en leur permettant de créer leurs propres réseaux d'amitié et de sociabilité en dehors de tout contrôle parental et social. Selon Hauk et Immordino (10), les parents et la société perdent de plus en plus le contrôle de leurs enfants sur le plan éducatif au profit des médias nationaux et internationaux qui diffusent souvent des valeurs qui sont aux antipodes des valeurs sociétales (rapports hommes et femmes, parents et enfants, etc.). Or, les médias prétendent à la fonction de socialisation de l'individu au même titre que les institutions comme la famille, l'école et la religion. Toutefois, les médias utilisent généralement des modes de transmissions des connaissances qui reproduisent des modèles culturels.

Depuis plusieurs années, notre pays se trouve confronté à de nombreux problèmes comportementaux des jeunes dans nos quartiers qui ne cadrent pas très souvent avec les valeurs sociales qui ont depuis longtemps régies nos sociétés. L'objectif était d'appréhender la place et le rôle de la télévision dans le processus de socialisation des jeunes au Mali.

\section{Méthodes}

II s'agissait d'une étude transversale descriptive qui s'est déroulée à Baco-djicoroni (Bamako) en février et mars 2016. Nous avons procédé à un échantillonnage aléatoire de 21 personnes dont 13 adolescents et 8 parents. Un échantillonnage raisonné a concerné quatre éducateurs de Baco-djicoroni, les responsables des émissions « Nous les enfants », « Maxi jeunes » et « Terroir » et le Directeur du programme de l'Office de Radiodiffusion Télévision du Mali (ORTM). Nous avons utilisé un questionnaire pour l'enquête en mode face à face. Le Logiciel Excel de Microsoft a été utilisé pour l'analyse quantitative. Les interviews de l'enquête qualitative ont été retranscrit verbatim et synthétisés en fonction des objectifs de l'étude.

Le consentement verbal libre et éclairé des enquêtés a été préalablement recueilli. L'anonymat et la confidentialité ont été garantis pour les données collectées au cours de l'étude.

\section{Résultats}

Deux parents (sur 8) et 9 adolescents (sur 13) étaient de sexe féminin. Les parents avaient majoritairement un âge compris entre 30 et 50 ans $(7 / 8)$ et un seul avait plus de 50 ans. Pour les adolescents, 11 avaient entre 12 et 15 ans.

Tous les parents sauf un et tous les adolescents ont affirmé disposé de télévision en famille.

La quasi-totalité des parents (7/8) regardaient la télévision avec leurs enfants. II en était de même pour les adolescents (12/13) qui regardaient la télévision avec leur famille.

Quatre parents et 10 adolescents ont affirmé regarder la télévision de temps en temps contre respectivement deux et un qui regardaient rarement et un et deux régulièrement. Cinq parents ont affirmé que leurs enfants regardaient la chaîne nationale ORTM. Les programmes qui les intéressaient étaient entre autres : dessins animés, Nous les enfants, maxi jeunes, Film Yèrèdombougou, Feuilleton et documentaire sur les animaux sauvages. Certains parents pensent que les programmes actuels des chaînes nationales ORTM et Africable peuvent contribuer à pérenniser nos valeurs culturelles.

$\mathrm{Au}$ total 12 adolescents ont affirmé connaître les programmes de l'ORTM dédiés aux jeunes. Parmi ceuxci, " Nous les enfants ", " Maxi jeunes », " Génération 21 ", "A l'écoute de l'école ", "Football " et "Yélèbougou » ont été les plus cités.

Entretien avec les spécialistes en science de l'éducation

En ce qui concerne les tendances actuelles de l'éducation, les spécialistes pensent que depuis l'avènement de l'école, il y a un recul de la conception traditionnelle de l'éducation. Le rôle de l'enseignant d'aujourd'hui se résume à l'instruction des enfants. Cet état de fait est 
exacerbé par l'influence de la rue, le non suivi des enfants à la maison et l'absence de cérémonie traditionnelle d'initiation.

Par rapport à la question de savoir s'il existe une différence entre les enfants d'hier et d'aujourd'hui, les spécialistes pensent à l'unanimité que les enfants ne sont pas fondamentalement différents mais ils subissent beaucoup l'influence des milieux d'évolution (école, internet).

D'après les spécialistes, les acquis culturels étaient transmis aux jeunes en famille et au sein de la communauté avant la télévision. Avec la télévision, il y a un véritable dilemme entre l'éducation africaine et celle occidentale pour les jeunes.

Les spécialistes de l'éducation interrogés n'ont pas un avis tranché par rapport à leur perception ou définition de la télévision, ils pensent qu'elle pourrait être soit un loisir, un moyen de communication rapide ou un auxiliaire pédagogique.

A la question de savoir si la télévision assure la continuité ou perturbe les mécanismes classiques de transmission de l'héritage culturel au Mali, la majorité pensent qu'elle la perturbe.

D'après les spécialistes, le rôle et la place de la télévision dans la socialisation de l'enfant seraient de compléter les acquis culturels ou de participer à leur culture générale grâce à la découverte d'autres horizons.

La majorité des spécialistes (3/4) pensent que les émissions réservées au jeune public ne répondent pas aux besoins de socialisation des enfants et des jeunes.

Entretien avec les responsables des émissions et programme de l'ORTM

L'un des objectifs de l'ORTM est de développer une politique de production et de diffusion des programmes Radio TV en rapport avec les objectifs de développement économique et culturel du pays au triple plan international, national, régional et local. A ce titre, son cahier de charge lui impose la sensibilisation des téléspectateurs.

Par rapport aux questions communes posées à tous nos interlocuteurs, il ressort que (i) les programmes visant à perpétuer les valeurs culturelles locales sont Terroir (cité par la majorité), Maxi jeunes, à l'écoute de l'école, MAGNAMAKA etc. Cependant, une personne pense qu'il n'y a pas de programme réel à cet effet ; (ii) la moitié des responsables interrogés pensent que la télévision assure la continuité des mécanismes classiques de transmission de l'héritage culturel au Mali.

\section{Discussion}

Dans notre étude, la majorité des parents avait un âge compris entre 30 et 50 ans, pour les adolescents il était majoritairement situé entre 12 et 15 ans.

Nous avons trouvé que la télévision était disponible dans toutes les familles des personnes interrogées (parents et adolescents) à l'exception d'un parent qui avait plus de 50 ans. La réception des programmes se faisait en famille pour la majorité des parents et des adolescents. Sanfo A. trouve un résultat similaire à Ouagadougou en 1995 (11).
En ce qui concerne la fréquence de réception des programmes, elle était majoritairement de temps en temps pour les enquêtés. Ce résultat est proche de celui Sanfo A. en 1995 où $63,7 \%$ regardaient avec moins d'assiduité la télévision (11). Cela pourrait s'expliquer par le fait que tous les membres ne sont pas intéressés par le même genre d'émissions.

La chaîne nationale ORTM était regardée par la majorité de leurs enfants d'après les parents. La multiplicité des programmes dédiés aux jeunes (dessins animés, Nous les enfants, maxi jeunes, Film Yèrèdombougou) pourrait expliquer cette situation. Le renforcement du contenu de ces programmes pourrait favoriser la transmission des valeurs culturelles.

Aussi, les adolescents interrogés connaissaient dans leur grande majorité, les programmes de l'ORTM dédiés aux jeunes. Cet état de fait pourrait contribuer à la transmission des valeurs locales contrairement aux chaînes étrangères.

L'interprétation des données des entretiens approfondis nous a permis de dégager deux positions essentielles : la première est constituée par les enquêtés qui soutiennent que la télévision nationale a une influence positive sur les jeunes de façon générale et en particulier sur les mécanismes de transmission des valeurs sociales, la deuxième est constituée par les enquêtés qui soutiennent le contraire.

\section{- L'influence positive}

A travers les interviews réalisés, il ressort que la télévision joue au Mali une fonction culturelle puissante et qu'elle diffuse des programmes essentiellement en direction des jeunes ce qui les permet de suivre de façon concrète à travers les images et les mots, les valeurs et les principes de la société.

L'existence de programmes visant à perpétuer les valeurs culturelles ancestrales maliennes a été mise en avant par la majorité de personnes interrogées. Cela pourrait expliquer le fait que la moitié de nos interlocuteurs pensaient que la télévision assurait la continuité des mécanismes classiques de transmission de l'héritage culturel.

Les éducateurs ont eu des positions souvent contradictoires. Un parmi les quatre éducateurs interrogés pense que ça ne perturbe pas à condition d'être mal exploité. Selon lui si les programmes sont bien coordonnés en fonction du temps et des moyens alors c'est un puissant moyen de transmission des acquis culturel.

Deux autres trouvent que la télévision est un moyen de socialisation des enfants car son rôle est la sensibilisation, la distraction et l'instruction. C'est le cas dans l'étude de Makwambeni et Salawu (12). Quand les enfants sont devant la télévision tout le monde est concentré, il n'y a pas de violence, il y'a un silence et c'est dans ce silence qu'ils s'aiment et se cultivent.

\section{- L'influence négative}

La majorité des éducateurs pensent que la télévision perturbe les mécanismes classiques de transmission de 
nos valeurs culturelles qui ont tendance à être oubliées au profit d'autres cultures diffusées à travers la télévision. Dans ces conditions, les enfants ont tendance à adopter les coutumes occidentales au dépend de celles africaines et surtout maliennes. Les tenants de cette position ont souligné que les jeunes n'exploitent que le côté négatif qui aboutit à l'acculturation et qui serait à l'origine du déclin de l'autorité parentale. Barkow et al. trouvent que les médias remplacent les parents et les enfants prennent comme modèle les acteurs surtout les célébrités (13). En Fiji, l'avènement de la télévision a impacté la recherche de nouvelles corpulences par les jeunes adolescentes (14). Ferguson trouve un résultat similaire (15). Les enquêtés ont aussi soutenu que si la télévision est bien, elle pourrait servir à la fois d'instrument pédagogique et informationnelle. Becker $A E$ soutient aussi que la télévision peut avoir un bon et un mauvais côté (14). Malheureusement, l'enfant regarde des images qu'il ne doit pas regarder.

Aussi les enquêtés ont souligné la part de responsabilité des parents dans la gestion des programmes télévisuels destinés aux jeunes. Les émissions regardées par les parents peuvent impacter celles des adolescents comme le démontre Edgerly et al. (16). Beaucoup de nos interlocuteurs, particulièrement les éducateurs, ont noté la démission des parents dans l'éducation des enfants, s'appuyant sur l'école. Hors, cette dernière n'assure de nos jours que la formation intellectuelle. Certains auteurs pensent que l'influence est beaucoup plus perceptive sur les familles pauvres (17). C'est pourquoi le temps passé devant l'écran est aussi souvent jugé comme facteur $(18,19)$ avec un impact sur le temps de sommeil $(20)$ et les risques de problèmes de santé (21-23).

II apparait donc que ce n'est pas la télévision en tant que tel qui perturbe, mais le contenu des programmes et des images qui sont diffusées et l'absence ou la faiblesse du contrôle parental sur ces derniers. Sinon, beaucoup de nos interviewés trouvent que la télévision peut être à la fois un excellent outil pédagogique, informationnel, éducatif et socialisateur.

\section{Conclusion}

La télévision joue un rôle beaucoup plus perturbateur dans la transmission de nos valeurs de société. Cependant, si des efforts sont faits pour la diversification et le renforcement des programmes dédiés aux jeunes, cette tendance pourra s'atténuer ou même s'inverser. II revient aux parents d'inciter leurs enfants à une utilisation judicieuse de ce moyen de communication. Une étude plus large pourra mieux appréhender ce phénomène.

\section{Références}

1. Castra M. Socialisation. Sociologie [Internet]. 1 août 2013 [cité 14 juin 2021]; Disponible sur: http://journals.openedition.org/sociologie/1992

2. Qribi A. Socialisation et identité. L'apport de Berger et Luckmann à travers « la construction sociale de la réalité ». Bull Psychol. 2010;Numéro 506(2):133-9.
3. Durkheim E. Éducation et Sociologie. Httplstliepiiep-UnescoorgcgiBinwwwi32exeinepidoc1int2000013016100. 1 janv 1999. 4. Little W. Chapter 5. Socialization. In: Introduction to Sociology - 1st Canadian Edition [Internet]. BCcampus; 2014 [cité 15 juin 2021]. Disponible sur:

https://opentextbc.ca/introductiontosociology/chapter/cha pter5-socialization/

5. OCTOBRE S. Intervention aux 7ème

Rencontres des services d'action éducative des bibliothèques, musées et archives, le 9 décembre 2009 à la BNF. Sources enquêtes Tic 2005, Insee. 2005.

6. McCoy D. The Impact of Socialization on Personality Formation and Gender Role Development. 1 janv 1990;

7. Atkin AL, Yoo HC. Familial racial-ethnic socialization of Multiracial American Youth: A systematic review of the literature with MultiCrit. Dev Rev. 1 sept 2019;53:100869.

8. Grusec JE. Socialization Processes in the Family: Social and Emotional Development | Annual Review of Psychology. Annu Rev Psychol.

2011;62:243-69.

9. définition de la télévision - Google Search [Internet]. [cité 14 juin 2021]. Disponible sur:

https://www.google.com/search?q=d\%C3\%A9finition+de $+\mid \mathrm{a}+\mathrm{t} \% \mathrm{C} 3 \% \mathrm{~A} 91 \% \mathrm{C} 3 \% \mathrm{~A} 9 \mathrm{vision} \& \mathrm{sxs}$ rf

10. Hauk E, Immordino G. Parents, Television and Cultural Change [Internet]. 2011 [cité 15 juin 2021].

Disponible sur:

http://digital.csic.es/bitstream/10261/35540/1/86811.pdf

11. Sanfo A. Télévision et socialisation de l'enfant dans la ville de Ouagadougou : relais ou perturbation des mécanismes classiques de transmission des acquis culturels [Internet] [Mémoire de Maîtrise].

[Ouagadougou]: Université de Ouagadougou; 1995 [cité 11 juill 2021]. Disponible sur:

https://www.yumpu.com/fr/document/read/12003003/ress ource

12. Makwambeni B, Salawu A. Accounting for youth audiences' resistances to HIV and AIDS messages in the television drama Tsha Tsha in South Africa. SAHARA J J Soc Asp HIVAIDS Res Alliance. 6 févr 2018;15(1):20-30.

13. Barkow JH, O'Gorman R, Rendell L. Are the New Mass Media Subverting Cultural Transmission? - , 2012. SAGE J. 2012;16(2):121-33.

14. Becker AE. Television, Disordered Eating, and Young Women in Fiji: Negotiating Body Image and Identity during Rapid Social Change. Cult Med Psychiatry. 1 déc 2004;28(4):533-59.

15. Ferguson CJ, Muñoz ME, Garza A, Galindo M. Concurrent and prospective analyses of peer, television and social media influences on body dissatisfaction, eating disorder symptoms and life satisfaction in adolescent girls. J Youth Adolesc. janv 2014;43(1):1-14. 
16. Edgerly S, Thorson K, Thorson E, Vraga EK, Bode L. Do parents still model news consumption? Socializing news use among adolescents in a multidevice world. New Media Soc. 1 avr 2018;20(4):1263-81. 17. Nguyen HV, Moschis GP, Shannon R. Effects of family structure and socialization on materialism: a life course study in Thailand. Int $\mathrm{J}$ Consum Stud. 2009;33(4):486-95.

18. Hardy LL, Baur LA, Garnett SP, Crawford D, Campbell KJ, Shrewsbury VA, et al. Family and home correlates of television viewing in 12-13 year old adolescents: The Nepean Study. Int J Behav Nutr Phys Act. 10 sept 2006;3:24.

19. Radesky JS, Christakis DA. Increased Screen Time: Implications for Early Childhood Development and Behavior. Pediatr Clin North Am. oct 2016;63(5):827-39.
20. Royant-Parola S, Londe V, Tréhout S, Hartley $\mathrm{S}$. The use of social media modifies teenagers' sleeprelated behavior. L'Encephale. sept 2018;44(4):321-8. 21. Browne KD, Hamilton-Giachritsis C. The influence of violent media on children and adolescents:a public-health approach. Lancet Lond Engl. 19 févr 2005;365(9460):702-10.

22. Saunders TJ, Vallance JK. Screen Time and Health Indicators Among Children and Youth: Current Evidence, Limitations and Future Directions. Appl Health Econ Health Policy. juin 2017;15(3):323-31.

23. Zdanowicz N, Reynaert C, Jacques D, Lepiece B, Dubois T. Screen Time and (Belgian) Teenagers. Psychiatr Danub. sept 2020;32(Suppl 1):36-41. 